\title{
A subsurface add-on for standard atomic force microscopes
}

G. J. Verbiest, D. J. van der Zalm, T. H. Oosterkamp, and M. J. Rost

Citation: Review of Scientific Instruments 86, 033704 (2015); doi: 10.1063/1.4915895

View online: http://dx.doi.org/10.1063/1.4915895

View Table of Contents: http://aip.scitation.org/toc/rsi/86/3

Published by the American Institute of Physics

\section{Articles you may be interested in}

Nanoscale subsurface imaging via resonant difference-frequency atomic force ultrasonic microscopy Journal of Applied Physics 101, 114324 (2007); 10.1063/1.2743908

Imaging of subsurface structures using atomic force acoustic microscopy at $\mathrm{GHz}$ frequencies Journal of Applied Physics 109, 084324 (2011); 10.1063/1.3573484

Ultrasound holography for noninvasive imaging of buried defects and interfaces for advanced interconnect architectures

Applied Physics Letters 95, 263101 (2009); 10.1063/1.3263716

Elastic phase response of silica nanoparticles buried in soft matter

Applied Physics Letters 93, 133113 (2008); 10.1063/1.2987460

Ultrasonic force microscopy for nanometer resolution subsurface imaging

Applied Physics Letters 64, 178 (1998); 10.1063/1.111524

Modeling the effect of subsurface interface defects on contact stiffness for ultrasonic atomic force microscopy Applied Physics Letters 84, 5368 (2004); 10.1063/1.1764941

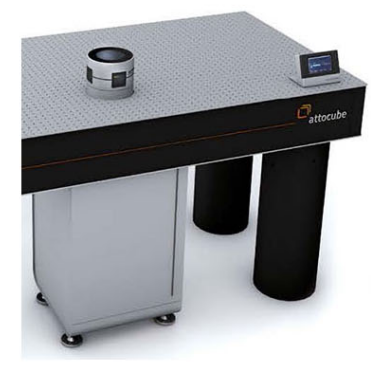

Obstruction free access

optical table with integrated cryocooler

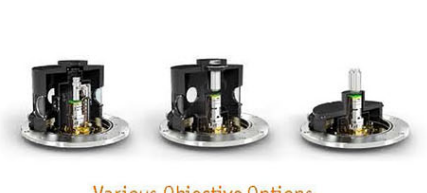

Various Objective Options

\section{attoDRY800}

- Cryogenic Temperatures

- Ultra-Low Vibration

- Optical Table Included

- Fast Cooldown

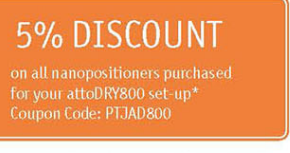




\title{
A subsurface add-on for standard atomic force microscopes
}

\author{
G. J. Verbiest, ${ }^{1, \text { a) }}$ D. J. van der Zalm, ${ }^{2}$ T. H. Oosterkamp, ${ }^{2}$ and M. J. Rost ${ }^{2, b)}$ \\ ${ }^{1}$ JARA-FIT and II. Institute of Physics, RWTH Aachen University, 52074 Aachen, Germany \\ ${ }^{2}$ Kamerlingh Onnes Laboratory, Leiden University, P.O. Box 9504, 2300 RA Leiden, The Netherlands
}

(Received 19 November 2014; accepted 9 March 2015; published online 26 March 2015)

\begin{abstract}
The application of ultrasound in an Atomic Force Microscope (AFM) gives access to subsurface information. However, no commercially AFM exists that is equipped with this technique. The main problems are the electronic crosstalk in the AFM setup and the insufficiently strong excitation of the cantilever at ultrasonic $(\mathrm{MHz})$ frequencies. In this paper, we describe the development of an add-on that provides a solution to these problems by using a special piezo element with a lowest resonance frequency of $2.5 \mathrm{MHz}$ and by separating the electronic connection for this high frequency piezo element from all other connections. In this sense, we support researches with the possibility to perform subsurface measurements with their existing AFMs and hopefully pave also the way for the development of a commercial AFM that is capable of imaging subsurface features with nanometer resolution. (C) 2015 AIP Publishing LLC. [http://dx.doi.org/10.1063/1.4915895]
\end{abstract}

\section{INTRODUCTION}

A general holy grail in microscopy is to nondestructively image the interior of a sample or, equivalently, to image below a surface. This goal is achieved by acoustic (ultrasound) microscopy, in oil field searching technology, by Magnetic Resonance Imaging (MRI), and in a Transmission Electron Microscope (TEM). However, except for a TEM, all these microscopes suffer from a relatively bad resolution. The latest generation of TEMs ${ }^{1}$ can clearly reach even sub- $\AA$ resolution, but the samples are limited in thickness, as the electron beam has to penetrate them. Moreover, these machines require ultrahigh vacuum such that imaging under ambient conditions is hardly possible and, in addition, the high-energy electron beam might also have an influence on the sample.

Consequently, it was great news ${ }^{2-4}$ that the combination of ultrasound with an AFM, a technique that is widely used in many different fields, ${ }^{5-11}$ also enables subsurface imaging, but now on the nanometer scale, on almost any type of sample, and in almost any condition.

By now, ultrasound-AFM techniques have been applied to image nanoparticles inside polymer matrices, ${ }^{4,12-16}$ voids in metals, ${ }^{17}$ the inside of mammal cells, ${ }^{4,18,19}$ the inside of plant cells, ${ }^{20}$ and for the determination of resonance frequencies as well as their mode shapes of nanomechanical systems. ${ }^{21}$ Although it has been successfully demonstrated on all these different systems that ultrasound-AFM provides access to otherwise unobtainable sample information, the interpretation of these images is not well understood, as the physical origin of the contrast formation as well as the incredible resolution remain (until now) a mystery. First steps towards a deeper understanding have been recently set, ${ }^{22-42}$ however, the real questions have not been answered yet.

Concerning the technical aspects, three (slightly) different modes have been developed that all enable subsurface infor-

\footnotetext{
a)Verbiest@physik.rwth-aachen.de

b)Rost@physics.leidenuniv.nl
}

mation with an AFM by applying ultrasound with a frequency in the order of a few MHz: in Ultrasonic Force Microscopy $(\mathrm{UFM})^{2,43}$ the ultrasonic wave is sent from the back through the sample, while in Waveguide-UFM ${ }^{44}$ the sound wave is sent through the cantilever. Heterodyne Force Microscopy (HFM) ${ }^{3}$ combines both techniques in one and reaches, as an advantage, a better time resolution, a higher signal-to-noise ratio, and (still under debate) also a better spatial resolution. Moreover, as HFM can also detect the phase between the two ultrasound signals (through the sample and the cantilever), it is thought to be the only technique that might deliver full 3D information: it should be possible to determine the depth of a feature from a 2D map of the phase difference measured on the sample surface.

A crucial requirement that enables indirectly the detection of the ultrasound in all these different ultrasonic AFM techniques is a real excitation of a low frequency signal in the cantilever's motion, which, therefore, usually lies in the $\mathrm{kHz}$ regime. It is exactly this motion that contains the subsurface information, as the cantilever is insensitive to the highfrequency ultrasonic excitation(s). UFM and Waveguide-UFM work with a low frequency amplitude modulation, whereas HFM works with a nonlinear coupling between the two ultrasonic sound waves to generate a low frequency excitation at their difference frequency that is explicitly tuned on (or below) the first resonance of the cantilever. ${ }^{26-28}$ It is the nonlinear interaction between the cantilever's tip and the sample that generates the low frequency signal in the cantilever's motion in all three ultrasound AFM techniques.

If one considers the, in general, rather extreme requirements of a standard AFM on the mechanical stability, vibration decoupling, and the prevention of electronic cross talk, one quickly realizes that the implementation of ultrasound is an issue both for the mechanical as well as the electrical stability of the microscope. In addition, the excitation by ultrasound needs to be effectively transmitted to the cantilever and/or the sample, such that both really oscillate (mechanically) at the ultrasonic frequency. To ensure stable measurements with 
high resolution, we, first, have to make sure that the ultrasound as well as the low frequency nonlinear signal does not significantly excite a vibration in the mechanical loop of the AFM, e.g., by exciting a resonance frequency. Second, we have to minimize the electronic crosstalk of the drive signals for the ultrasound, as it otherwise would limit the applicable frequency range of the ultrasound..$^{51}$ We are not aware of any commercially available AFM setup that meets these requirements and, therefore, we needed to develop our own AFM add-on.

In this paper, we present an AFM-head with a specialized cantilever holder that meets all of the requirements mentioned above. To show the applicability of our design to any commercially available AFM setup, and to get a quick start with our research, we designed the head for a Nanoscope $\mathrm{V}$ of Bruker $^{45}$ existing in our laboratory. The manuscript is organized as follows. First, we review some of the basic concepts of an AFM that are important to understand the rest of the paper. Second, we provide the background information of the electronic crosstalk that arises when the cantilever is ultrasonically excited. Third, we introduce our design of an AFM head that solves not only the electronic crosstalk but also the stability and the excitation problem. Then, we measure and analyze the characteristics of the piezo elements that are used for the ultrasound excitation of the cantilever, before we finally characterize the performance of our cantilever holder (AFM-head) and compare it with the commercially available one.

\section{BASICS OF AN AFM}

A typical AFM setup is shown in Fig. 1. The bending of the cantilever is commonly measured via an optical beam (red), which is reflected from the back of the cantilever onto a photodetector. Even a small change in bending of the cantilever, caused by a height variation of the surface, results in a measurable signal on the photodetector (blue).

The photodetector is not only sensitive to a small change in bending of the cantilever, but it also detects any (harmonic) motion of the cantilever, which is induced with a piezo element

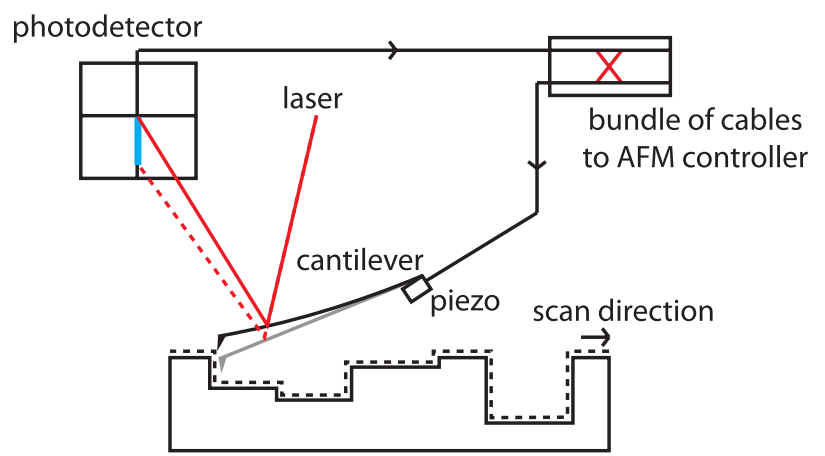

FIG. 1. Schematics of an AFM setup: The bending of the cantilever is measured via an optical beam method (red). A height change of the surface results in a change in bending of the cantilever, which leads to a different signal of the photodetector (indicated with the dashed red line and the blue line). The piezo element is used to harmonically excite the cantilever. Note that the photodetector signal and the drive signal of the piezo element go through the same bundle of cables. This results in crosstalk at ultrasonic frequencies (indicated by the red cross). that is usually mounted just below the cantilever. It is important to note that the drive signal of this piezo element and the signal of the photodetector go through the same bundle of cables of the AFM hardware. This naturally results in crosstalk between these two signals, as indicated with a red cross in Fig. 1.

\section{ELECTRONIC CROSSTALK}

Let us consider two parallel cables in the used AFM setup to illustrate the electronic crosstalk problem. The diameter of these cables is typically $400 \mu \mathrm{m}$, while the separation between them is typically $400 \mu \mathrm{m}$. The connection between the AFM setup and the control electronics is at least $2 \mathrm{~m}$, which results in a total capacitance of approximately $70 \mathrm{pF}$ (assuming a dielectric with $\epsilon_{r}=4$ between the cables). Together with an output/input resistance of $50 \Omega$, this results in an electronic high pass filter between the cables with a pass frequency of $55 \mathrm{MHz}$. For a higher output/input resistance, this pass frequency will be even lower. As a consequence, approximately $5 \%$ of the ultrasonic drive voltage at $3 \mathrm{MHz}$ is directly transmitted into the other cable. A serious problem arises, if this cable is used for the electronic signal of the cantilever's motion, as it limits the applicable ultrasound to low ultrasonic frequencies. The reason for this is that the crosstalk seemingly generates a cantilever motion, even if there is no cantilever mounted. This crosstalk can be even so severe that the electronically introduced signal is larger than the voltage that corresponds to the real cantilever motion, if excited at these high, ultrasonic frequencies. In our case, we measured an apparent signal $V_{0}$ of the cantilever motion of $10 \mathrm{mV}$ (with mounted cantilever), when we applied a drive signal $V_{i}$ of $1 \mathrm{~V}$ at a frequency of $3 \mathrm{MHz}$. This $10 \mathrm{mV}$ limits our capability to measure the cantilever motion as we expect to receive a photodetecor signal of $3 \mathrm{mV}$ for the cantilever vibrating at $3 \mathrm{MHz}$. A real measurement is impossible with such a huge crosstalk.

The red line in Fig. 2 shows the frequency dependent transfer function $\left|V_{0} / V_{i}\right|$ of the cantilever, measured as $V_{0}$ of the photodetector signal, if we excite the cantilever with $V_{i}=1 \mathrm{~V}$ via the piezo element. To provide information about the signal-to-noise in our system, we measured also the spectral photodetector background (shown in black). The spectrum shows four resonances of the cantilever at $71 \mathrm{kHz}, 432 \mathrm{kHz}$, $1.183 \mathrm{MHz}$, and $2.277 \mathrm{MHz}$, which is in perfect agreement with the specs of an AC240 cantilever ${ }^{46}$ that we used in this measurement. Above the fourth resonance frequency of the cantilever, the photodetector signal mainly shows the crosstalk as discussed above, which is indeed approximately $5 \%$ of the drive amplitude. This also prevents us from observing the fifth and higher resonance frequencies of the cantilever.

A careful look to the spectrum of Fig. 2 reveals also a second problem. Whereas the detected amplitude of the first and second resonances of the cantilever are approximately equal, we clearly observe a decreased amplitude of the third resonance and an even smaller amplitude of the fourth resonance. This low-pass behavior indicates, in comparison to the used ultrasound, a rather low resonance frequency of the piezo element that is used to excite the cantilever. 


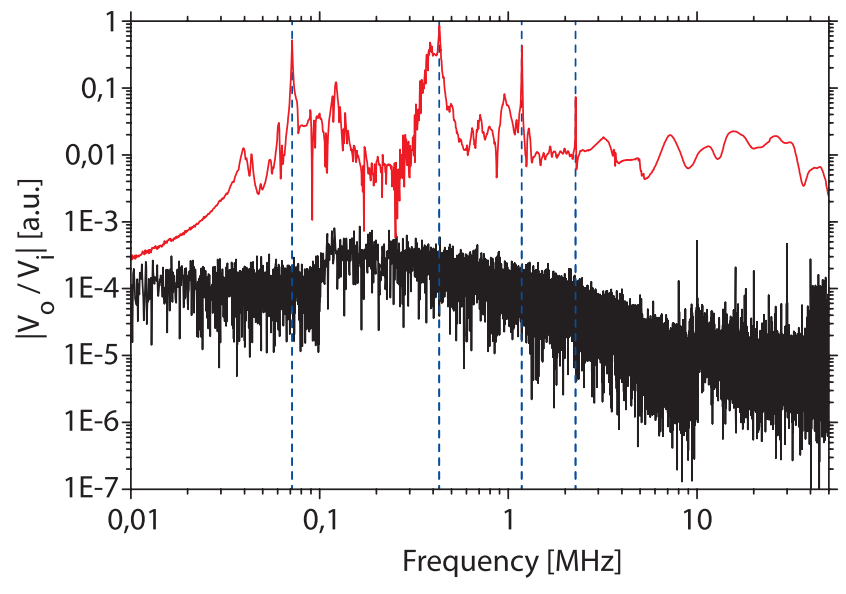

FIG. 2. Cantilever transfer function: The normalized amplitude $\left|V_{0} / V_{i}\right|$ (red) of the photodetector signal, if the cantilever is excited via the piezo element that we drive with $V_{i}=1 \mathrm{~V}$. To give an idea about the signal-to-noise ratio, we show in black the background noise of the photodetector. The vertical dashed lines indicate the resonance frequencies of the cantilever at $71 \mathrm{kHz}$, $432 \mathrm{kHz}, 1.183 \mathrm{MHz}$, and 2.277 MHz. Above the fourth resonance of the cantilever, the photodetector signal is mainly determined by the electronic crosstalk, which is also the reason that higher resonances frequencies can not be observed.

Both the crosstalk and the low resonance frequency of the piezo element prevented us from performing subsurface, ultrasound AFM experiments in our commercially available AFM.

\section{HOME-BUILT CANTILEVER HOLDER}

To meet the requirements, we had to solve the problems discussed in Sec. III, which resulted in a completely home-built cantilever holder as shown in Fig. 3. We solved the crosstalk by fully separating the drive signal of the piezo element from the electronic wiring of the photodetector. An additional improvement in terms of decoupling could be achieved by placing an electric ground just below the piezo element that excites the cantilever at ultrasonic frequencies. To address the excitation problem, we used a special piezo element with a free resonance frequency of approximately $4 \mathrm{MHz}$. With these improvements,

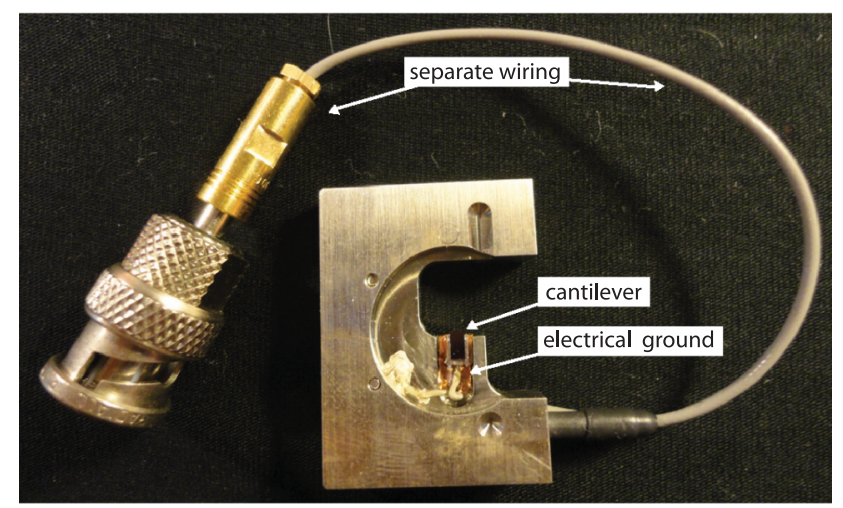

FIG. 3. Home-built holder: The electronic drive signal for the ultrasound is sent through a completely separate wiring. The electric ground that we added reduces the electronic crosstalk in the home-built holder. we are able to detect even the 7 th resonance frequency of the cantilever instead of only up to the 4 th (see Sec. VI).

As the details of the design of the piezo stack underneath the cantilever is crucial for the performance of the complete holder, we will in the following explain the important issues. The holder is depicted in (Fig. 4(a)) and the individual elements of the piezo stack are shown in Figs. 4(b) and 4(c).

For our design, we took the following considerations into account. First, we decided to use two separate piezo elements: one for the ultrasonic excitation of the cantilever (HF piezo element) and another for the relatively low-frequency tapping mode operation (LF piezo element). Second, to achieve an as high as possible lowest resonance frequency of the HF piezo element, as little as possible mass should be added, which has consequences for the isolations and electrodes. Third, both an electric ground and isolation plates are a prerequisite to reduce the electronic crosstalk within the stack as well as the coupling to the ultrasonic piezo element that excites the sample.

We decided to use isolation plates made of $\mathrm{Al}_{2} \mathrm{O}_{3}(0.2 \mathrm{~mm}$ thick) and special electrodes $(0.1 \mathrm{~mm}$ thick $\mathrm{Cu}$ foil) with an isolating varnish on one side. Although it might seem, in the following description, that we use the $\mathrm{Al}_{2} \mathrm{O}_{3}$ plates in a standard way simply for isolation purposes, the main application of these plates is the reduction of the electronic crosstalk between different electrodes by decreasing the involved capacity due to an increase of the distance between the electrodes. The application of the very special electrodes made it possible to reduce the total mass of the stack, which is beneficial for the resonance frequencies of both piezo elements (and therefore the transfer function of the cantilever). Unfortunately, the $\mathrm{Al}_{2} \mathrm{O}_{3}$ plates lower the resonance frequencies of both piezo elements significantly such that a proper trade-off between the electrical decoupling and the lowering of the frequencies had to be made.

In the final design, the cantilever (1) is glued to a titanium alignment chip (2), which has an angle of $13^{\circ}$ such that only the tip, and not the whole cantilever, touches the sample in the approached state. Directly assembled below is an $\mathrm{Al}_{2} \mathrm{O}_{3}$ plate (3) to reduce the electronic crosstalk between the HF and the sample piezo elements. We use the combination of electrode (4), HF piezo element (5), and electrode (6) for the ultrasonic excitation of the cantilever. Below electrode (6), there is an $\mathrm{Al}_{2} \mathrm{O}_{3}$ plate (7) and an electric ground (8) to reduce the electronic crosstalk within the entire stack. The electric ground is a large shield that is connected to the ground/mass of the cantilever holder: as it covers perfectly the entire groove as well as the LF piezo element, it works like a Faraday cage. This completely decouples electronically the HF from the LF piezo element. We use the combination of electrode (9), LF piezo element (10), and electrode (11) for the standard tapping mode operation with frequencies up to $\sim 400 \mathrm{kHz} .{ }^{52}$ The holder, on which the stack is mounted, is indicated with (12) and the electrical connections of the individual elements are provided in (d).

As the ultrasound attenuation in the $\mathrm{Al}_{2} \mathrm{O}_{3}$ plates attenuates $\sim 1.5 \mathrm{~dB} / \mathrm{cm}$ for low $\mathrm{MHz}$ frequencies, one looses $\sim 1.4 \%$ of the amplitude before the wave reaches the cantilever, if using the LF piezo element for the ultrasonic excitation. For the HF piezo element, this is only $0.7 \%$. In addition, the 


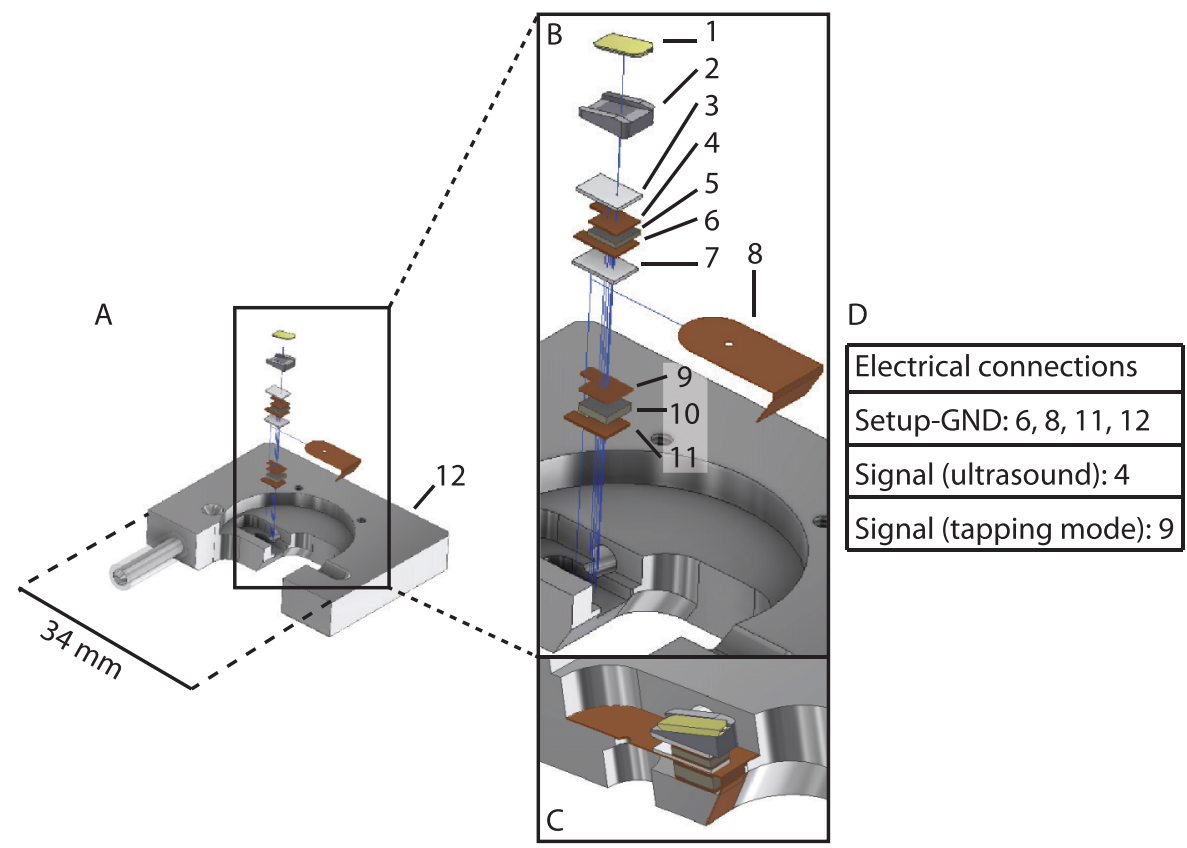

FIG. 4. Design of our home-built cantilever holder: The complete holder is depicted in (a) and a zoom-in, which shows the individual elements of the piezo stack, is provided in (b). A cross section through this stack is shown in (c). We used $0.2 \mathrm{~mm}$ thick isolation plates made of $\mathrm{Al}_{2} \mathrm{O}_{3}$ to reduce the electronic crosstalks. For the electrodes, we used $0.1 \mathrm{~mm}$ thick $\mathrm{Cu}$ foils with an isolating varnish on one side. The cantilever (1) is glued on a titanium alignment chip (2). Directly assembled below is an $\mathrm{Al}_{2} \mathrm{O}_{3}$ plate (3) to reduce the electronic crosstalk between the $\mathrm{HF}$ and the sample piezo elements. We use the combination of electrode (4), HF piezo element (5), and electrode (6) for the ultrasonic excitation of the cantilever. Below electrode (6), there is an $\mathrm{Al}_{2} \mathrm{O}_{3}$ plate (7) and an electric ground (8) that works like a Faraday cage, to reduce the capacitive coupling between the two piezo elements in the stack. We use the combination of electrode (9), LF piezo element (10), and electrode (11) for normal tapping mode operation. The holder, on which the stack is mounted, is indicated with (12) and the electrical connections are provided in (d).

amplitude at the cantilever is further reduced, as (due to the additional mass) the cut-off frequency of the low-pass filter is significantly lower for the LF than for the HF piezo element. Although the ultrasound attenuation in the $\mathrm{Cu}$ electrodes is larger $(\sim 15 \mathrm{~dB} / \mathrm{cm})$, the total thickness of the $\mathrm{Cu}$ foils is so small that they attenuate the amplitude with less than $6.7 \%$ for the LF and less than $1.7 \%$ for the HF piezo element. Due to the above described facts, it is favorable to apply the ultrasound for the cantilever by the HF piezo element and use the LF piezo element for, e.g., tapping mode operation. Although we expected that the used glue also strongly attenuates the ultrasonic wave, we obtain a significantly strong transmission to the cantilever, especially for the HF piezo element, as shown in Sec. IV.

For the assembly, we first glued all electrodes to the piezo elements using an electrical conducting epoxy (Epotek H20E) that we cured afterwards for $1 \mathrm{~h}$ at $120^{\circ} \mathrm{C}$. Then, the complete stack was glued together with an electrical nonconducting epoxy (Epotek H70E) followed by an annealing for 1 hour at $90^{\circ} \mathrm{C}$. We applied an external pre-load of $\sim 15 \mathrm{~N}$ during all gluing and curing steps.

From experience during operation, we later noticed that the noise of the measured amplitude and phase of the cantilever's motion could be significantly reduced by connecting also electrode (6) on purpose directly to the ground/mass of the cantilever holder. This "shortcut" makes the ground of the cantilever holder and the ground of the ultrasonic drive signal exactly the same. The ground of the drive electronics comes, therefore, from the ground of the cantilever holder (without a ground loop being present via the power supplies).
Since the LF piezo element is only operated at relatively low frequencies, the isolating varnish on the electrodes together with the nonconducting epoxy is sufficient to isolate it from the rest of the holder.

\section{PIEZO STACK CHARACTERIZATION}

To enable the application of ultrasonic frequencies as high as possible, we chose for special piezo elements with significantly high free resonance frequencies. Such piezo elements are commercially available from Applied Laser Technology $(\mathrm{ALT})^{47}$ and PI Ceramics ${ }^{48}$ and we decided to use the elements as characterized in Table I to excite the cantilever as well as the sample at ultrasonic frequencies. In order to receive optimal transmission of the piezo vibrations into both the cantilever and the sample, we directly glued them onto their corresponding piezo elements using Crystalbond 509.49

To check whether the first free resonance of the piezo element for the ultrasonic cantilever excitation is in the order

TABLE I. The used piezo elements: The table provides the dimensions, length $\times$ width $\times$ height $(\mathrm{L} \times \mathrm{W} \times \mathrm{H})$, and the material of the piezo elements that we applied to excite both the cantilever and the sample at ultrasonic frequencies.

\begin{tabular}{lcc}
\hline \hline & $\begin{array}{c}\text { Dimensions } \\
\text { Piezo }\end{array}$ & $\mathrm{L} \times \mathrm{W} \times \mathrm{H}(\mathrm{mm})$ \\
\hline Cantilever & $2 \times 2 \times 0.3$ & Material \\
Sample & $10 \times 10 \times 0.5$ & PIC $155^{48}$ \\
\hline \hline
\end{tabular}


of a few $\mathrm{MHz}$, we measured the voltage $V_{0}$ in the electric circuit shown in Fig. 5 as a function of frequency. The applied voltage $V_{i}$ was set to $1 \mathrm{~V}$. The equivalent electrical diagram of a piezo element is given by its static capacitance $C_{p}$ in combination with parallel RLC-circuits, which describe the mechanical resonance frequencies, due to the inherent mechanicalelectrical coupling given by the piezoelectric material. In Fig. 5, we only add one RLC-circuit, as we are only interested in the first mechanical resonance frequency of the piezo. The effective electrical impedance of the piezo element $Z_{\text {piezo }}$ is then

$Z_{\text {piezo }}(\omega)=\frac{1}{j \omega C_{p}}\left\{\frac{1+j \omega R_{r} C_{r}-\omega^{2} L_{r} C_{r}}{1+C_{r} / C_{p}+j \omega R_{r} C_{r}-\omega^{2} L_{r} C_{r}}\right\}$,

in which $\omega$ is the angular frequency of the applied voltage $V_{i}$. If one plots $\left|Z_{\text {piezo }}(\omega)\right|$ as a function of $\omega$, one first observes a minimum in the impedance (maximum in admittance), which is followed by a maximum in the impedance (minimum in admittance). The minimum in the impedance corresponds with the mechanical resonance frequency and occurs at $\omega=$ $1 / \sqrt{L_{r} C_{r}}$, whereas the maximum in the impedance is called the antiresonance at frequency $1 / \sqrt{L_{r}\left(C_{r}^{-1}+C_{p}^{-1}\right)^{-1}}$. For the measured voltage $V_{0}$ in the electric circuit shown in Fig. 5, we find

$$
\frac{V_{0}}{V_{i}}=\frac{R_{0}}{Z_{\text {piezo }}(\omega)+R_{0}} .
$$

For frequencies well below $1 / 2 \pi R_{0} C_{p}$, Eq. (2) reduces to

$$
\frac{V_{0}}{V_{i}}=R_{0} Z_{\text {piezo }}^{-1} .
$$

As we measure the root-mean-square value $\left|V_{0}\right|$ and use the ingoing amplitude $V_{i}$, we also take the absolute value of the right side of Eq. (3) and divide it by $\sqrt{2}$,

$$
\left|\frac{V_{0}}{V_{i}}\right|=\frac{R_{0} C_{p} / \sqrt{2}}{1+C_{r} / C_{p}} \sqrt{\frac{\left(1-\left(\frac{\omega}{\omega_{a r}}\right)^{2}\right)^{2}+\left(\frac{\omega}{\omega_{a r} Q_{a r}}\right)^{2}}{\left(1-\left(\frac{\omega}{\omega_{r}}\right)^{2}\right)^{2}+\left(\frac{\omega}{\omega_{r} Q_{r}}\right)^{2}}},
$$

in which $\omega_{a r}\left(\omega_{r}\right)$ is the antiresonance (resonance) frequency and $Q_{a r}\left(Q_{r}\right)$ is the corresponding quality factor. The antiresonance frequency $\omega_{a r}$ and its corresponding quality factor $Q_{a r}$ is equivalent to the quality factor of the RLC-circuit and therefore given by

$$
\omega_{a r}=1 / \sqrt{L_{r} C_{r}}, \quad Q_{a r}=\frac{1}{R_{r}} \sqrt{\frac{L_{r}}{C_{r}}} .
$$

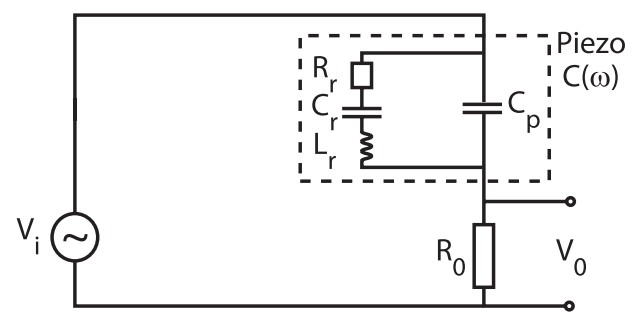

FIG. 5. Electric circuit used to determine the resonance frequencies of the piezo elements: The applied voltage $V_{i}$ is set to $1 \mathrm{~V}$. We measured the voltage $V_{0}$ over a low Ohmic resistance of $R_{0}=1 \Omega$, to determine the capacitance $C(\omega)$ as a function of the excitation frequency.
Similar, we can express the resonance frequency $\omega_{r}$ and its corresponding quality factor $Q_{r}$ in terms of the parameters of the RLC-circuit:

$$
\omega_{r}=\omega_{a r} \sqrt{1+C_{r} / C_{p}}, \quad Q_{r}=Q_{a r}\left(1+C_{r} / C_{p}\right) .
$$

Let us now compare the characteristics of the two piezo elements in our cantilever holder. Figures 6 and 7 show the absolute value of the transfer function $\left|V_{0} / V_{i}\right|$ as a function of frequency for the LF and the HF piezo element, respectively. The insets show the complete measured frequency range from $1 \mathrm{kHz}$ to $50 \mathrm{MHz}$, while the main graphs provide a zoom around the first resonance frequency. The measurement is shown in black, the RLC-circuit (Eq. (4)) is shown in red, and the dashed red line shows the transfer function of the capacitance $C_{p}$ in series with the resistance $R_{0}$.

For the LF piezo element, we extract a mechanical resonance frequency of $0.923 \mathrm{MHz}$ and an antiresonance at $0.948 \mathrm{MHz}$, of which the corresponding quality factors are 10 and 11, respectively. These numbers have to be compared with those of the HF piezo element, for which we found a resonance frequency of $2.543 \mathrm{MHz}$ and an antiresonance at $2.552 \mathrm{MHz}$, as well as the corresponding quality factors of 13 and 15 , respectively. From the measured resonance and antiresonance frequencies, we conclude that the HF piezo element should be able to excite the cantilever up to $2.5 \mathrm{MHz}$. The resonance frequency of the LF piezo element is more than a factor 2 smaller, which indicates that it is not possible to excite the cantilever at $\mathrm{MHz}$ frequencies with this piezo element.

From the linear slopes in Figs. 6 and 7, we determine the capacitances $C_{p}$ of both the LF and the HF piezo element. The LF piezo element has a $C_{p}$ of $2.1 \mathrm{nF}$ and that of the HF piezo element is $1.4 \mathrm{nF}$. We compare these numbers with the values calculated for a parallel plate capacitor with the dimensions given in Table I. We calculate a $C_{p}$ of $2.6 \mathrm{nF}$ and $0.2 \mathrm{nF}$ for the LF piezo element and the HF piezo element, respectively. These values are of the same order of magnitude as the measured ones with a larger deviation for HF piezo element. A reason for this could be an additional parallel capacitance, e.g., due to the connecting cables.

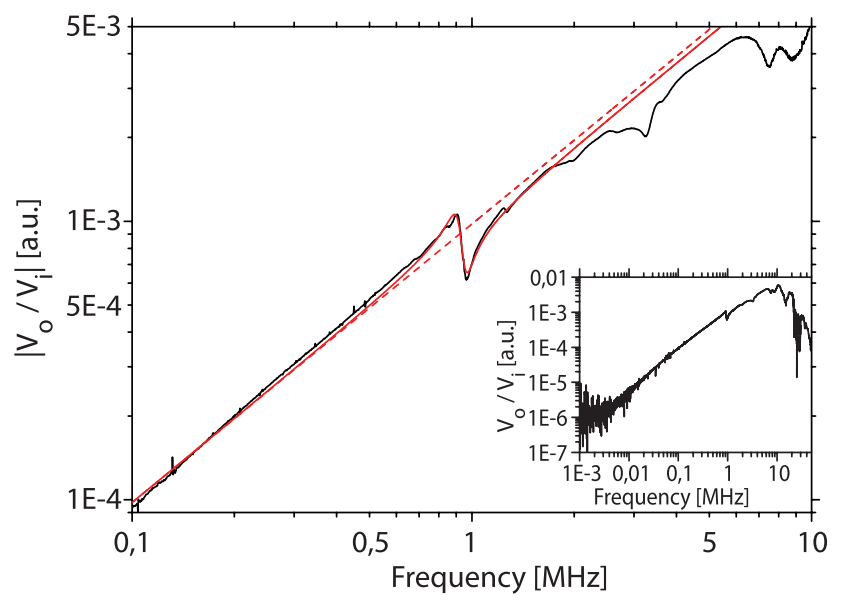

FIG. 6. Transfer function $\left|V_{0} / V_{i}\right|$ as a function of frequency for the LF piezo element: the inset shows the complete frequency range and the main graph is a zoom around the first resonance frequency $(\sim 0.9 \mathrm{MHz})$. The figure shows the measurement (black), the RLC-circuit (Eq. (4)) (red), and the transfer function of a high pass filter (dashed, red). 


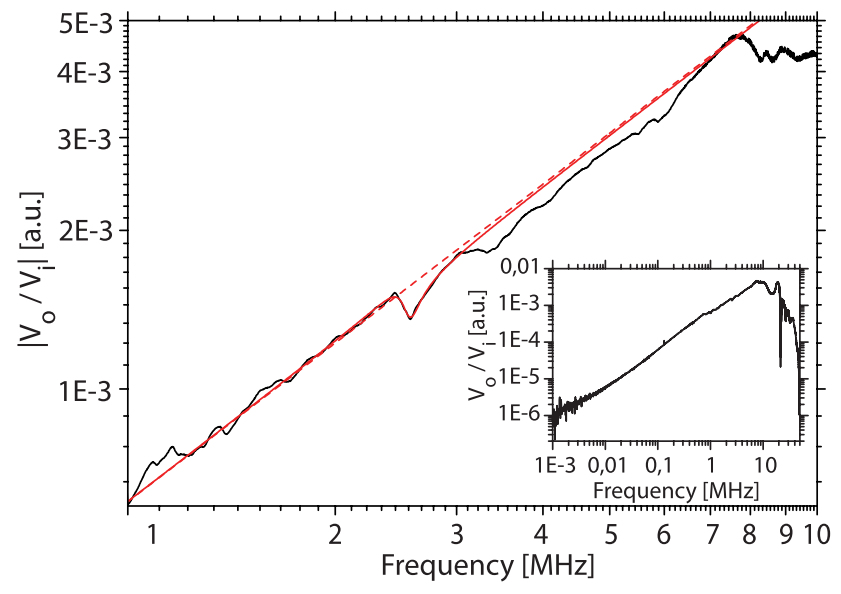

FIG. 7. Transfer function $\left|V_{0} / V_{i}\right|$ as a function of frequency for the HF piezo element: the inset shows the complete frequency range and the main graph is a zoom around the first resonance frequency $(\sim 2.5 \mathrm{MHz})$. The figure shows the measurement (black), the RLC-circuit (Eq. (4)) (red), and the transfer function of a high pass filter (dashed, red).

Most ultrasound AFM techniques also need an ultrasound excitation of the sample. By using a piezo element similar to the one used for the ultrasound excitation of the cantilever, see Table I, it is also possible to significantly excite the sample at $\mathrm{MHz}$ frequencies.

\section{PERFORMANCE OF OUR HOLDER}

To illustrate the final performance of our cantilever holder, we characterized the photodetector signal while driving the cantilever one time via the LF and the other time via the HF piezo element. To be fully comparable with the earlier measurements shown in Fig. 2, we used not only exactly the same cantilever but also the same parameters. Moreover, to demonstrate the reduction in crosstalk, we also measured the photodiode signal while driving the HF piezo element without a mounted cantilever. The results are shown in Fig. 8.

Let us start with the background signal (here purple, whereas it is black in Fig. 2) of the photodetector, which means that there is no cantilever mounted and none of the piezo elements is driven with an electronic signal. This signal is fully unchanged, which is no surprise without alterations in this electronic path. Although not shown in Fig. 2, here we show (in blue) also the photodetector background signal, if we apply an electronic signal with $1 \mathrm{~V}$ to the HF piezo element to provide information about the signal-to-noise level for the final application. Up to $300 \mathrm{kHz}$, this noise level follows the background noise level of the photodetector. At around $1 \mathrm{MHz}$, we find an electronic resonance, after which the crosstalk reduces to a level of $2 \times 10^{-4} V_{i}$. The improvement is significant, as this noise level is $\sim 2$ orders of magnitude lower than the noise above $2 \mathrm{MHz}$ in the original head. Obviously, the "old" high noise level for high frequencies is present (see red line), if we electronically drive the LF piezo element in our new holder. The reason for this is that the cabling of the LF piezo element still goes through the AFM head and, therefore, has a large crosstalk with the photodetector signal.

The transfer function of the cantilever, measured via the photodetector signal, is shown in red for the case that we

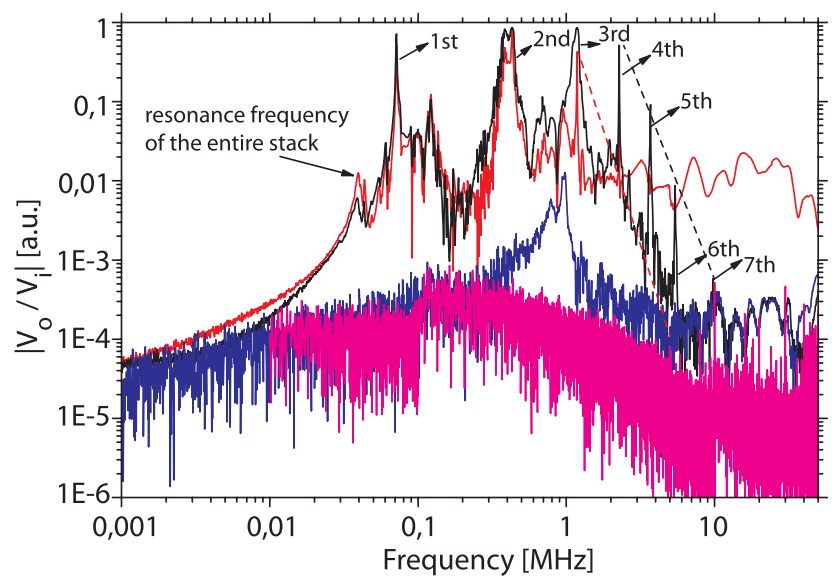

FIG. 8. Cantilever Transfer Function of the New Holder. Excited with $V_{i}=1$ $\mathrm{V}$ via the HF piezo element (black) and via the LF piezo element (red). Using the HF piezo element, we can observe the motion up the 7th resonance frequency of the cantilever at of $\sim 10 \mathrm{MHz}$, while it was possible only up to $\sim 2 \mathrm{MHz}$ (4th resonance) with the original holder, which is comparable with the excitation via the LF piezo element. Please note also that the noise above $2 \mathrm{MHz}$ is significantly decreased. The dashed lines indicate the low-pass filter action of the piezo elements above their resonance frequencies. To give an idea about the signal-to-noise ratio, we show the background noise of the photodetector without a mounted cantilever: in purple without any drive signal and in blue, if we apply the drive signal to the HF piezo element.

excite the cantilever via the LF piezo element, and in black, if we excite it via the HF piezo element. There is a significant improvement, as we now can resolve even the 7th resonance frequency, whereas we could only work up to the 4th one with the original microscope. Ultrasonic cantilever excitations are now possible and detectable up to $\sim 10 \mathrm{MHz}$, whereas we were limited to $\sim 2 \mathrm{MHz}$ before. This is a huge improvement in SubSurface-AFM, as both the contrast and the resolution scale with the frequency squared. ${ }^{22}$

In addition, one notices a clear difference between the excitation via the LF versus the HF piezo element. Both piezo

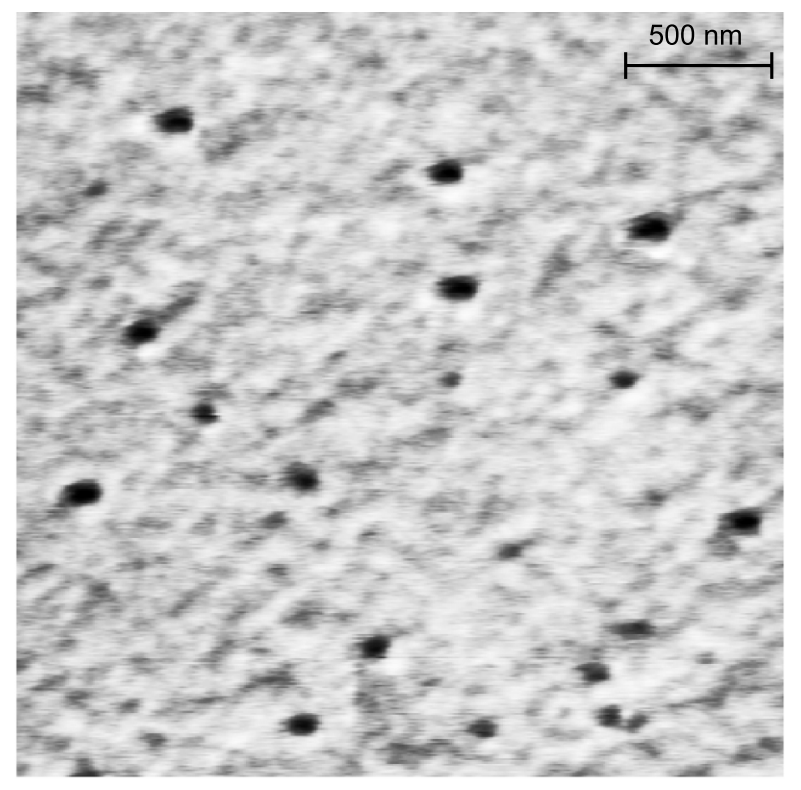

FIG. 9. Subsurface-AFM experiment: The black "spots" are $20 \mathrm{~nm}$ large gold particles that we carefully embedded $80 \mathrm{~nm}$ below the surface. 
elements have their own lowest resonance frequency, and as the LF piezo element has more mass on top, its resonance frequency is lower. Beyond the resonance frequency the mechanical amplitude dramatically decreases, since the system acts a mechanical low-pass filter. This behavior can clearly be seen at the red and the black dashed lines that connect the maxima of the resonance peaks for one particular choice of a piezo element. The filter effect sets in around $1 \mathrm{MHz}$ for the LF piezo element and around $2.5 \mathrm{MHz}$ for the $\mathrm{HF}$ piezo element, which are both perfectly in agreement with the piezo elements characterization described in Sec. V. This again shows the importance of choosing a design and a piezo element such that the final resonance frequency is as high as possible.

Finally, to demonstrate the functionality of our holder, we performed a SubSurface-AFM experiment. ${ }^{29}$ For this, we prepared a model sample that contained $20 \mathrm{~nm}$ large gold nanoparticles embedded in a $200 \mathrm{~nm}$ thick polymer film such that the particles are all buried 80 nm's deep. In the experiment, we used our special holder to excite the cantilever at $2.5 \mathrm{MHz}$. Using a similar piezo element, the sample was excited at $2.52 \mathrm{MHz}$. Figure 9 shows the measured amplitude of the heterodyne difference frequency signal, which clearly shows the buried gold nanoparticles as black spots. This proves the functionality of our holder for SubSurface-AFM experiments.

\section{SUMMARY}

We showed that one should address two main general issues when designing an AFM cantilever holder that is suited for ultrasonic applications. First, due to the high frequencies, electronic crosstalk between the cables of the drive signal to the piezo element that excite the cantilever and the cable of the photodetector output signal is a general concern at ultrasonic frequencies. Second, to reach sufficient excitation amplitudes of the cantilever, conventional piezo elements with resonance frequencies below the desired excitation frequency are far from adequate. One needs not only to design a holder with low mass as possible on top of the piezo element that is used to excite the cantilever at ultrasonic frequencies, but one also needs to make use of special piezo elements with natural high resonance frequencies.

We solved these problems by using a piezo element with a resonance frequency of $2.5 \mathrm{MHz}$ in combination with the physical separation of the electronic drive signal of this piezo element from the entire AFM. As a result, we were able to excite the cantilever up to its seventh resonance frequency, which is approximately at $10 \mathrm{MHz}$. In comparison to the original holder, this breaks down to an improvement of a factor 4 in frequency range, which delivered an increase in subsurface resolution as well as in contrast with a factor of $16 .^{22}$ This allowed us to gather significant insight in the general technical aspects of HFMs ${ }^{26-28}$ as well as in the determination of the physical mechanism that is responsible for the contrast formation! ${ }^{29}$

By applying a similar piezo element mounted underneath the sample, we were also able to transmit sufficiently strong ultrasound waves into the sample such that we easily could measure the ultrasonic motion of $\sim 3 \AA$ at the sample surface. ${ }^{27}$
Our solution allows researchers to build their own HFM add-on such that they can cheaply and quickly perform subsurface measurements with existing AFM's in their labs. This might also pave the way for commercial applications of ultrasound AFM techniques. Ultimately, this is of crucial importance, as subsurface imaging is a long standing desire in microscopy in general. One might think about applications in cell biology, ${ }^{4,13,18-20}$ material science, ${ }^{12,14,15,29}$ and nanoelectronics $^{4,17}$ as well as nanomechanics. ${ }^{21}$

\section{ACKNOWLEDGMENTS}

The research described in this paper has been performed under and financed by the NIMIC ${ }^{50}$ consortium under project 4.4.

T. H. Oosterkamp acknowledges support from an ERC starting grant.

${ }^{1}$ http://www.fei.com.

${ }^{2}$ O. Kolosov and K. Yamanaka, Jpn. J. Appl. Phys. 32, 1095 (1993).

${ }^{3}$ M. T. Cuberes, H. E. Assender, G. A. D. Briggs, and O. V. Kolosov, J. Phys. D: Appl. Phys. 33, 2347 (2000)

${ }^{4}$ G. S. Shekhawat and V. P. Dravid, Science 310, 89 (2005).

${ }^{5}$ D. Rugar and P. Hansma, Phys. Today 43(10), 23 (1990).

${ }^{6}$ N. Jalili and K. Laxminarayana, Mechatronics 14, 907 (2004).

${ }^{7}$ H.-J. Butt, B. Capella, and M. Cappl, Surf. Sci. Rep. 59, 1 (2005).

${ }^{8}$ P. Hansma et al., Appl. Phys. Lett. 64, 1738 (1994).

${ }^{9}$ F. J. Giessibl, Phys. Rev. B 56, 24 (1997).

${ }^{10}$ J. W. M. Frenken, T. H. Oosterkamp, B. L. M. Hendriksen, and M. J. Rost, Mater. Today 8, 5 (2005).

${ }^{11}$ F. C. Tabak et al., Ultramicroscopy 110, 599 (2010).

${ }^{12}$ S. A. Cantrell, J. H. Cantrell, and P. T. Lillehei, J. Appl. Phys. 101, 114324 (2007).

${ }^{13}$ L. Tetard et al., Appl. Phys. Lett. 93, 133113 (2008).

${ }^{14}$ M. Cuberes, J. Nanomater. 2009, 762016.

${ }^{15}$ K. Kimura, K. Kobayashi, K. Matsushige, and H. Yamada, Ultramicroscopy 133, 41 (2013).

${ }^{16}$ R. Garcia and E. T. Herruzo, Nat. Nanotechnol. 7, 217 (2012).

${ }^{17}$ G. S. Shekhawat, A. Srivastava, S. Avasthy, and V. P. Dravid, Appl. Phys. Lett. 95, 263101 (2009).

${ }^{18}$ L. Tetard, A. Passian, R. H. Farahi, and T. Thundat, Ultramicroscopy 110, 586 (2010).

${ }^{19}$ L. Tetard et al., Nat. Nanotechnol. 3, 501 (2008).

${ }^{20}$ L. Tetard et al., Ultramicroscopy 110, 701 (2010).

${ }^{21}$ D. Garcia-Sanchez, A. M. van der Zande, A. San Paulo, B. Lassagne, P. L. McEuen, and A. Bachtold, Nano Lett. 8(5), 1399-1403 (2008).

${ }^{22}$ G. J. Verbiest, J. N. Simon, T. H. Oosterkamp, and M. J. Rost, Nanotechnology 23, 145704 (2012).

${ }^{23}$ Z. Parlak and F. L. Degertekin, J. Appl. Phys. 103, 114910 (2008).

${ }^{24}$ A. F. Sarioglu, A. Atalar, and F. L. Degertekin, Appl. Phys. Lett. 84, 5368 (2004).

${ }^{25}$ L. Tetard, A. Passian, and T. Thundat, Nat. Nanotechnol. 5, 105 (2010).

${ }^{26}$ G. J. Verbiest, T. H. Oosterkamp, and M. J. Rost, Ultramicroscopy 135, 113 (2013).

${ }^{27}$ G. J. Verbiest, T. H. Oosterkamp, and M. J. Rost, Nanotechnology 24, 365701 (2013).

${ }^{28}$ G. J. Verbiest and M. J. Rost, Nat. Commun. 6, 6444 (2015).

${ }^{29}$ G. J. Verbiest, T. H. Oosterkamp, and M. J. Rost, e-print arXiv:1307. 1292.

${ }^{30}$ K. Yamanaka, H. Ogiso, and O. Kolosov, Jpn. J. Appl. Phys. 33, 3197 (1994).

${ }^{31}$ K. Yamanaka et al., Surf. Interface Anal. 27, 600 (1999).

${ }^{32}$ K. Yamanaka, Y. Maruyama, T. Tsuji, and K. Nakamoto, Appl. Phys. Lett. 78, 13 (2001).

${ }^{33}$ J. A. Turner, S. Hirsekorn, U. Rabe, and W. Arnold, J. Appl. Phys. 82, 3 (1997).

${ }^{34}$ T. R. Rodriguez and R. Garcia, Appl. Phys. Lett. 84, 3 (2004).

${ }^{35}$ U. Rabe, J. Turner, and W. Arnold, Appl. Phys. A 66, S277 (1998).

${ }^{36}$ U. Rabe et al., Ultrasonics 38, 430 (2000).

${ }^{37}$ J. L. Bosse, P. D. Tovee, B. D. Huey, and O. V. Kolosov, J. Appl. Phys. 115, 144304 (2014). 
${ }^{38}$ F. Dinelli, S. K. Biswas, G. A. D. Briggs, and O. V. Kolosov, Phys. Rev. B 61, 20 (2000).

${ }^{39}$ E. T. Herruzo and R. Garcia, Beilstein J. Nanotechnol. 3, 198-206 (2012).

${ }^{40}$ S. Hirsekorn, U. Rabe, and W. Arnold, Nanotechnology 8, 57 (1997).

${ }^{41}$ S. Hirsekorn, Appl. Phys. A 66, S249 (1998).

${ }^{42}$ J. R. Lozano and R. Garcia, Phys. Rev. B 79, 014110 (2009).

${ }^{43}$ O. V. Kolosov and G. A. D. Briggs, patent WO 98/08046 (1998).

${ }^{44}$ K. Yamanaka and S. Nakano, Jpn. J. Appl. Phys. 35, 3787 (1996)

${ }^{45}$ http://www.bruker.com.
${ }^{46} \mathrm{http}: / /$ probe.olympus-global.com/en/.

${ }^{47}$ http://www.alt.com.

${ }^{48} \mathrm{http}: / /$ www.piceramic.com.

${ }^{49} \mathrm{http}: / / \mathrm{www} \cdot$ aremco.com

${ }^{50} \mathrm{http}: / /$ www.realnano.nl.

${ }^{51}$ Note that the subsurface contrast increases with the square of the ultrasonic frequency, ${ }^{22}$ but that the electronic cross talk is also significantly enhanced at higher frequencies.

${ }^{52}$ The Nanoscopes are capable to provide a tapping mode excitation up to $\sim 5 \mathrm{MHz}$. 